\title{
Image Enhancement of Laser Welding Molten Pool Feature in Frequency Domain
}

\author{
Ling $\mathrm{MO}^{\mathrm{a}}$, Xiangdong $\mathrm{GAO}^{\mathrm{b}, *}$, Deyong $\mathrm{YOU}^{\mathrm{b}}$ \\ School of Electromechanical Engineering, Guangdong University of Technology, No.100 West \\ Waihuan Road, Higher Education Mega Center, Panyu District, Guangzhou 510006, China \\ a ling-mo@163.com, ${ }^{b}$ gaoxd666@126.com, *Corresponding author
}

Keywords: molten pool, keyhole, frequency domain, image enhancement

\begin{abstract}
Molten pool image feature extraction is the key to judge welding quality. The commonly used method of spatial domain image processing is an operation for each pixel of an image, which is sensitive to noises. The frequency domain image processing could overcome the shortcomings of the spatial domain image process, it modifies the parameters within the image transform domain and operates the whole image, and then implements inverse transformation to get processed image. During the high-power fiber laser welding, in order to obtain effective and accurate molten pool keyhole image features, frequency domain image enhancement was used to remove noises and obtain the molten pool keyhole feature information. The experimental results showed that the image enhancement in the frequency domain was better than the spatial domain image processing in molten pool feature extraction.
\end{abstract}

\section{Introduction}

With the appearance of high power laser, laser welding has been widely used in the machinery, automobile, shipbuilding, aerospace, steel and other industrial fields. High power fiber laser welding has apparent characteristics such as high energy density, rapid welding speed, severe temperature change [1]. With the rapid development of vision sensors, the welding quality can be judged by analyzing the molten pool images. However, it is difficult to get clear images in actual industrial welding environment, the useful information should be extracted from molten pool images[2]. Image enhancement technology could highlight important information in an image and weaken or remove unwanted information. Ambiguous image could be converted into a clear image with large amount of useful imformation, while the image noises are removed, the image edge and some ROI (region of interested) are enhanced. Also, through image enhancement, it simplifies the interested target detection. Image enhancement can be divided into the spatial domain and frequency domain enhancement. Spatial enhancement is aimed directly at each pixel of the image by gray level transformation to extend the gray level contrast and smoothing. It is also an edge enhancement and sharpening technology to operate the image. The frequency domain image enhancement transforms the original image from spatial domain to frequency domain, using FFT, DCT, wavelet analysis methods to process the image and obtain the results through inverse transformation. Here, in order to remove noises and improve image quality, two low frequency pass filters and one high frequency pass filter were used to suppress the noise frequency components and pass the molten pool useful frequenct component, then through IFT to obtain the filtered images and achieve the purpose of image smoothing.

\section{Experimental setup}

The experimental setup consisted of an IPG Photonics YLR-1000 high-power fiber laser welding equipment (laser power 10kW), shielding gas (argon), a Panasonic VR-016 type 6-axis robot and a welding experimental platform equipped with a high speed camera, motor servo and fixing devices [3]. The schematic drawing of the whole experiment setup is shown in Fig.1. To eliminate the interference and obtain near infrared images of a molten pool, a combination filtering system with a 
filter length of 960-990nm was placed in front of the vision sensor. Molten pool image recordings were carried out at 1000 frames/s by a $512 \times 512$ pixel high speed NAC camera. The used material was a Type 304 stainless steel plate with dimensional size of $150 \mathrm{~mm} \times 100 \mathrm{~mm} \times 10 \mathrm{~mm}$, and the seam gap was less than $0.1 \mathrm{~mm}$. The welding speed was $2.5 \mathrm{~m} / \mathrm{min}$.

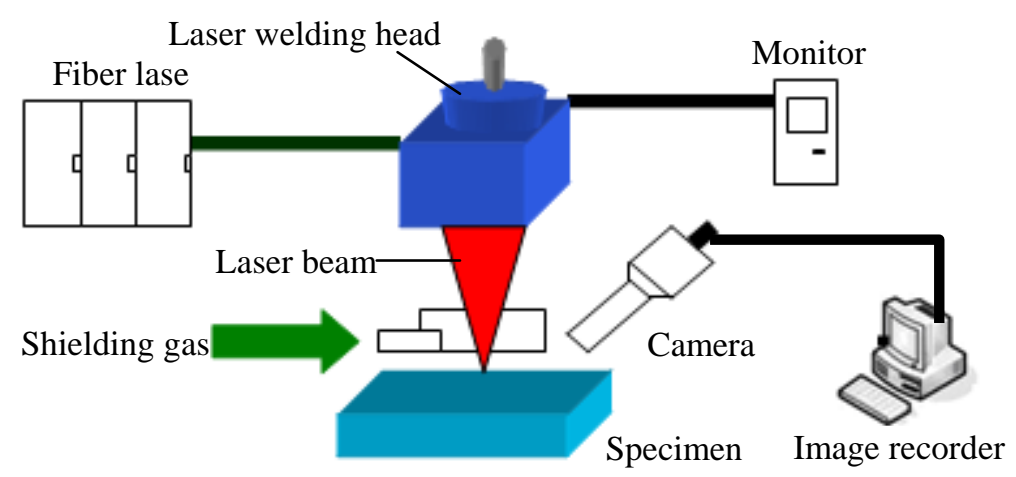

Fig.1 Schematic of fiber laser welding experimental setup

\section{Welding experiments and analysis}

Image pre-processing. Fig. 2 shows the original image, which includes a molten pool, keyhole and weld seam. In the welding process, fiber laser beam was focused on the seam, the metal material adjacented to the seam melted into liquid and formed a molten pool. The temperature on the liquid metal material was higher than the solid parts. Keyhole is the characteristic of deep pentration laser welding, it was formed by the high-energy-density laser beams. Fig.3 shows the gray level distribution curve, we could see three peaks in the curve, reflecting the different parts of an welding image. The first peak cooresponds to the background of the image, most parts are at low gray level because the temperature of the solid metal material is low. The second peaks cooresponds to the molten pool, this area is the second hot region. The third peak reflects the keyhole parts, which is the highest temperature position, so the gray level is close to 256. The original image needs to be processed to reduce the distortion. Median filter was applied to the original image, the basic priciple of the median filter is using the median pixel around a point to replace the point pixel value, making the pixel value close to the ture value, thereby eliminating isolated noise points. The filtered image is shown in Fig.4.

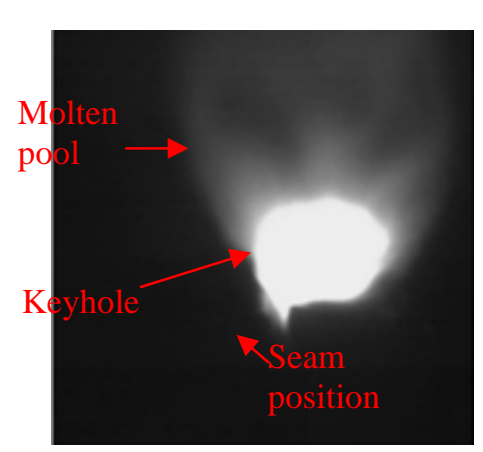

Fig.2 Original image

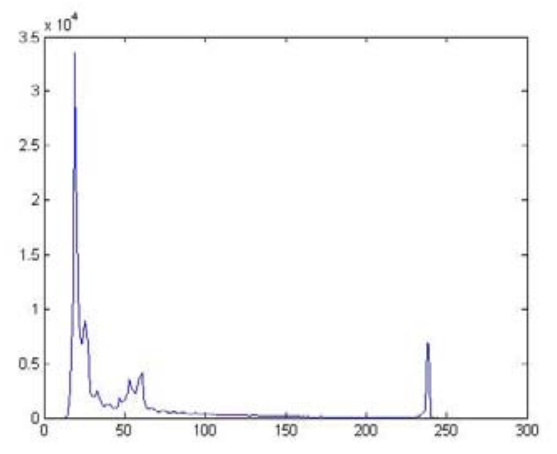

Fig.3 Gray level distribution curve

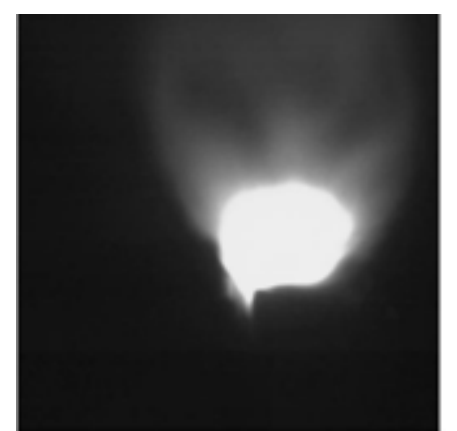

Fig.4 Image after Median filter

Image enhancement in frequency domain. In fact, digital image is a two-dimensional discrete fuction, we could use FFT to transform the image from spatial domain into the frequency domain. To analyze the frequency distribution of a molten pool image, Fig. 5 shows the frequency spectrum of the original image through FFT[4]. There is only a tiny spot in the middle of the picture. To observe the frequency spectrum clearly, we did the Logarithm operation after FFT, Fig. 6 presents the clear spectrum, it is a symmetric picture, most of the image energy concentrated on low frequency and 
median frequency segments, and the information of ROI was covered by the noises. The noises were mainly at high frequency segment.

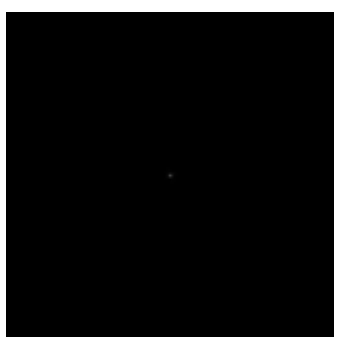

Fig.5 Fequency spectrum after FFT Fig.6 Fequency spectrum after FFT and

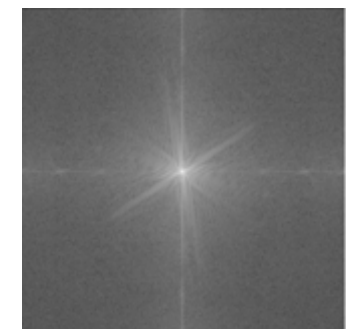

Logarithm

\section{Frequency domain filter}

Low-pass filter. Two low-pass filters were established to pass the low frequency components effectively. By the convolution theory, the low-pass filter mathematical experssion is : $G(u, v)=$ $\mathrm{F}(u, v) \mathrm{H}(u, v)$, where $\mathrm{F}(u, v)$ is the original image with noise in Fourier transform domain, and the $\mathrm{H}(u, v)$ is a transfer function, $\mathrm{G}(u, v)$ is an output image after low-pass filter in Fourier domain[5]. Fig.7 presents an image after ideal low pass filter. The ideal low-pass filter transform function is:

$$
H(u, v)= \begin{cases}1 & D(u, v) \leq D_{0} \\ 0 & D(u, v)>D_{0}\end{cases}
$$

where $\mathrm{D}_{0}$ is the cutoff frequency of the distance between the ideal low pass filter and the origin. $\mathrm{D}(u, v)$ is the distance between point $(u, v)$ to origin.

Fig.8 shows an image after Butterworth low pass filter, the function of Butterworth low pass filter can be depicted as:

$$
H(u, v)=\frac{1}{1+\left[\frac{D(u, v)}{D_{0}}\right]^{2 n}}
$$

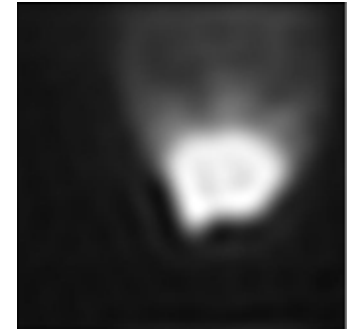

Fig.7 Pool image after ideal low pass filter
Fig.8 Pool image after Butterworth low pass filter High-pass filter. Because the image detail part cooresponded to the high frequency compoents, the high pass filter could be used to sharpen the image and protrude the keyhole area. High-pass filter is opposite to the low-pass filter, it makes the high frequency compents pass and weaken the low frequency. An image after high pass filter is shown in Fig.9. The molten pool and the background are groomy while the keyhole edge is clear to distiguish from the pool and background. High pass filter could highlight the edge of keyhole information and enhance the countour feature. Fig.10 shows the image after high pass filter and high frequency enhancement. The molten pool, keyhole and the seam position tip are all clear to observe.
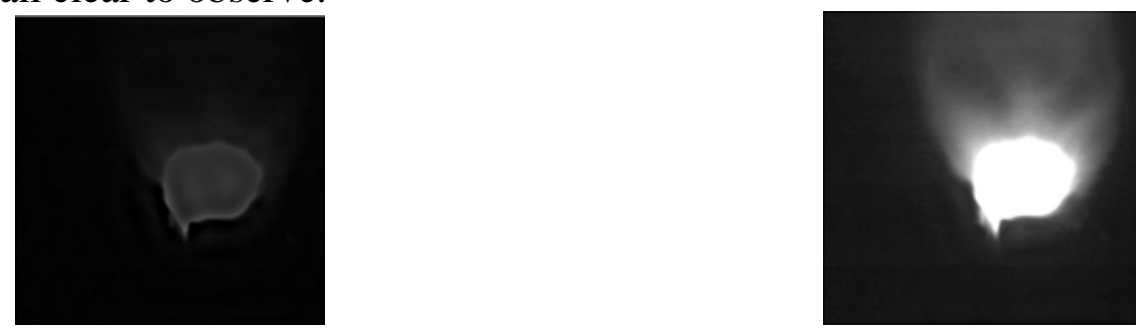

Published by Atlantis Press, Paris, France. (C) the authors 
Fig.9 Pool image after high pass filter Fig.10 High pass filter and high frequency enhancement

Weld pool keyhole feature extraction. Canny operator was used to obtain the keyhole edge after high pass filter and high frequency enhancement, which is shown in Fig.11. In contrast to the edge after spatial median filter in Fig.12, the former is more accurate than the latter one.

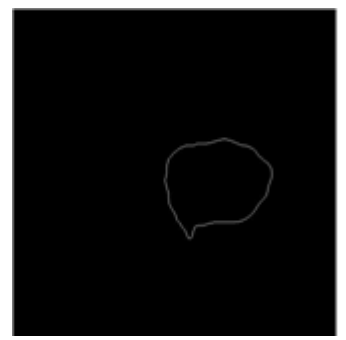

Fig.11 Canny edge detection after frequency domain enhancement

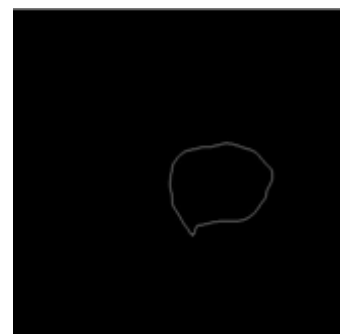

Fig.12 Canny edge detection after spatial domain enhancement

\section{Conclusions}

Using frequency domain enhancement to process the weld infrared image could obtain the molten pool information. Ideal low pass filter and Butterworth low pass filter were used in experiment, for the ideal low pass filter, different filter radius could obtain different filter results. The performance of Butterworth low pass filter was obviously better than the ideal low pass filter. An ideal high pass filter was used to highlight the keyhole and molten pool. Also, the keyhole edge could be extracted from a molten pool. Frequency domain filter could get more accurate keyhole contour.

\section{Acknowledgements}

This work was partly supported by the National Natural Science Foundation of China(51175095), the Guangdong Province Natural Science Foundation of China (10251009001000001, 9151009001000020) and the Specialized Research Fund for the Doctoral Program of Higher Education of China (20104420110001). Many thanks are given to Katayama Labotory of Osaka University, for their assistance of laser welding experiments.

\section{References}

[1] S. Katayama,Y. Kawahito and M.Mizutani: Physics Procedia Vol.5(2010), P.9.

[2] Gao J Q, Qin G L, Yang J L, He J G, Zhang T and Wu C S:Image processing of weld pool and keyhole in Nd: YAG laser welding based on edge predicting. //1st Sino-German Workshop on Simulation of Welding, 25-29 Oct. Berlin(2010),p.67

[3] Gao X D, You D Y,Seiji Katayama and Y. Kawahito, in: Proceedings of LAMP 2009-the 5th International Congresson Laser Advanced Materials Processing, Japan, \#129(2009), P.9

[4] Gao X D, Zhao C M, Bai T X and Ding D K: Transactions of the China welding institution. Vol.29(8)(2008), p.13

[5] Gonzales R C, Woods R E and Steven L.Eddins: Digital Image Using MATLAB.(Publishing House of Electronics Industry, Beijing 2004). 